\title{
Do Patients Lose Weight After Joint Arthroplasty Surgery? A Systematic Review
}

\author{
Maria C. S. Inacio MS, Donna Kritz-Silverstein PhD, \\ Elizabeth W. Paxton MA, Donald C. Fithian MD
}

Received: 4 April 2012/Accepted: 2 August 2012/Published online: 7 September 2012

(C) The Association of Bone and Joint Surgeons (B) 2012

\begin{abstract}
Background The ability of patients with a total joint arthroplasty (TJA) to lose weight after surgery has been investigated in a few studies with inconsistent results.

Questions/Purposes We asked: (1) What is the quality of evidence of current published literature on postoperative weight trends for patients who have had a TJA? (2) Do patients lose any weight after TJA? (3) Do patients lose a clinically meaningful amount of weight after TJA?
\end{abstract}

Each author certifies that he or she, or a member of his or her immediate family, has no funding or commercial associations (eg, consultancies, stock ownership, equity interest, patent/licensing arrangements, etc) that might pose a conflict of interest in connection with the submitted article.

All ICMJE Conflict of Interest Forms for authors and Clinical Orthopaedics and Related Research editors and board members are on file with the publication and can be viewed on request. This work was performed in the Joint Doctoral Program in Epidemiology at San Diego State University/University of California, San Diego, San Diego, CA, USA

M. C. S. Inacio ( $\square)$

Joint Doctoral Program in Epidemiology, San Diego

State University/University of California,

3033 Bunker Hill St., San Diego, CA 92109, USA

e-mail: maria.cs.inacio@kp.org

D. Kritz-Silverstein

Department of Family and Preventive Medicine,

University of California San Diego School

of Medicine, San Diego, CA, USA

E. W. Paxton

Surgical Outcomes and Analysis Department,

Kaiser Permanente, San Diego, CA, USA

D. C. Fithian

Department of Orthopaedic Surgery, Southern California

Permanente Medical Group, El Cajon, CA, USA
Methods We conducted a systematic review of PubMed and the Cochrane Library. Studies were summarized according to the Preferred Reporting Items for Systematic Reviews and Meta-analyses Statement. Studies were reviewed for quality of evidence and limitations according to the Grading of Recommendations Assessment, Development, and Evaluation (GRADE) criteria. Twelve studies were identified, one case-cohort study and 11 case series. Most studies were from single-surgeon or single-hospital series. Five studies included THAs and TKAs, four only THAs, and three only TKAs. We determined study type, level of evidence, inclusion criteria, procedures, proportion of patients who changed weight, body composition assessment, time of composition assessment, statistical analysis performed, and subgroup analysis conducted.

Results Owing to the observational nature of the studies and the serious limitations identified, all were considered very low quality according to GRADE criteria. Studies reported $14 \%$ to $49 \%$ of patients had some weight loss at least 1 year postoperatively.

Conclusions We found no conclusive evidence that weight or body composition increases, decreases, or remains the same after TJA.

\section{Introduction}

Concurrent with the increased prevalence of total joint arthroplasty (TJA) [6, 9, 19, 20, 33], the prevalence of comorbid conditions among patients having these procedures is increasing $[8,25]$. Obesity is one comorbidity that not only increases the risk of TJA [7, 13, 16] but also increases the risk of revision [32], surgical site infection [21, 29], dislocation [30], and thromboembolic events [23]. In addition, obesity is associated with a greater likelihood 
of having bilateral TKAs [24, 35]. If this modifiable risk factor were eliminated, a considerable portion of TJAs in patients in the United States likely could be delayed or prevented, and complications associated with these procedures could be reduced.

The ability of a patient with a TJA to lose weight postoperatively is unclear. Some studies show patients' weight and/or BMI after surgery continues to be elevated, despite successful recovery from surgery, regain of biomechanical strength, increase in functional status [11, 12, $31]$, relief of pain [11, 12], and an increase in overall quality of life [12]. Other studies suggest a proportion of patients either do not change or lose some of their excess weight $[1,2,11,12,18,22,34,37]$. Some of the inconsistency in the previous literature may be attributable to variation in inclusion criteria in the samples studied, measurement of the main outcome (weight, BMI, body composition change) methodology (some are patient reported, others determined by a blinded trained researcher), and analysis conducted. Understanding the sample characteristics in each study is important for interpretation and generalizability of the results. For example, inclusion of patients with rheumatoid arthritis, who have different recovery and postoperative challenges, is likely to confound the outcome studied. Similarly, the inclusion of revision arthroplasties or hip resurfacing procedures introduces bias to the assessments, as patients undergoing these procedures have profiles different from those of patients needing a primary THA. Finally, the inclusion of patients with bilateral procedures to the analysis could confound the findings of the studies because patients undergoing bilateral procedures are typically younger and have better general health status in the case of knee arthroplasties or special circumstances in the case of hip arthroplasties, which are important to take into consideration. Moreover, not only is the methodology heterogeneous, the overall quality of the studies is often poor, making it difficult to generalize their results.

We therefore asked: (1) Using the Grading of Recommendations Assessment, Development and Evaluation (GRADE), what is the quality of evidence of current published literature on patients postoperative weight trends after TJA? (2) Overall, do patients who have TJA lose any weight after surgery? (3) Using the FDA definition for clinically meaningful weight loss (body weight change of $5 \%$ or greater), do patients lose weight after TJA?

\section{Search Strategy and Criteria}

The first author (MCSI) conducted a comprehensive systematic review of citation databases PubMed and Cochrane Library. Articles from 1990 to 2011 were searched for the key words: "weight change", "BMI change", "obesity", and "obesity change" in combination with the words "arthroplasty" or "joint replacement". The only limits to the electronic search applied were that studies had to be in English and had to report on an adult sample (18 years or older). In addition, references in the full articles identified were reviewed for additional candidate articles. We identified 498 articles in the PubMed database, none in the Cochrane Library, and two from references in reviewed articles (Fig. 1).

The first author (MCSI) performed a manual review of the abstracts of the 500 studies to identify all studies evaluating weight or BMI change in a sample undergoing TJAs. All studies on the topic were eligible for inclusion; no studies were excluded based on level of evidence, study design, exclusion and inclusion criteria, followup time, attrition, or obvious selection or informational bias. This inclusive approach was followed to evaluate the quality of the literature on this topic and current findings.

After initial review, we identified 12 studies (Fig. 1). Studies were summarized according to the Preferred Reporting Items for Systematic Reviews and Meta-analyses (PRISMA) Statement [27]. PRISMA recommends use of a flow of information to illustrate the review process and a 27 -item checklist that should be included in a systematic review. All articles were reviewed and the following data extracted: type of study, level of evidence, inclusion and exclusion criteria (diagnosis for procedure, types of procedure, bilateral assessment), main exposure of interest (TJA or THA and TKA), main outcome of interest (proportion of patients who lost weight, gained, or remained unchanged), body composition assessment (weight or BMI), time of outcome assessment, statistical analysis performed, and subgroup analysis (Table 1).

The first author (MCSI) further reviewed the studies to assess the quality of evidence and limitations (risk of bias)

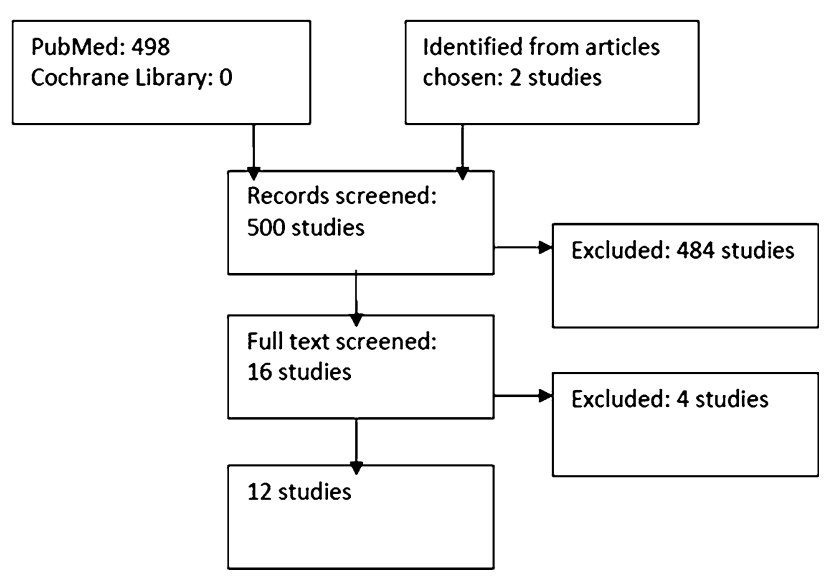

Fig. 1 The flow of information for identification of studies on weight trends after TJA is shown. 


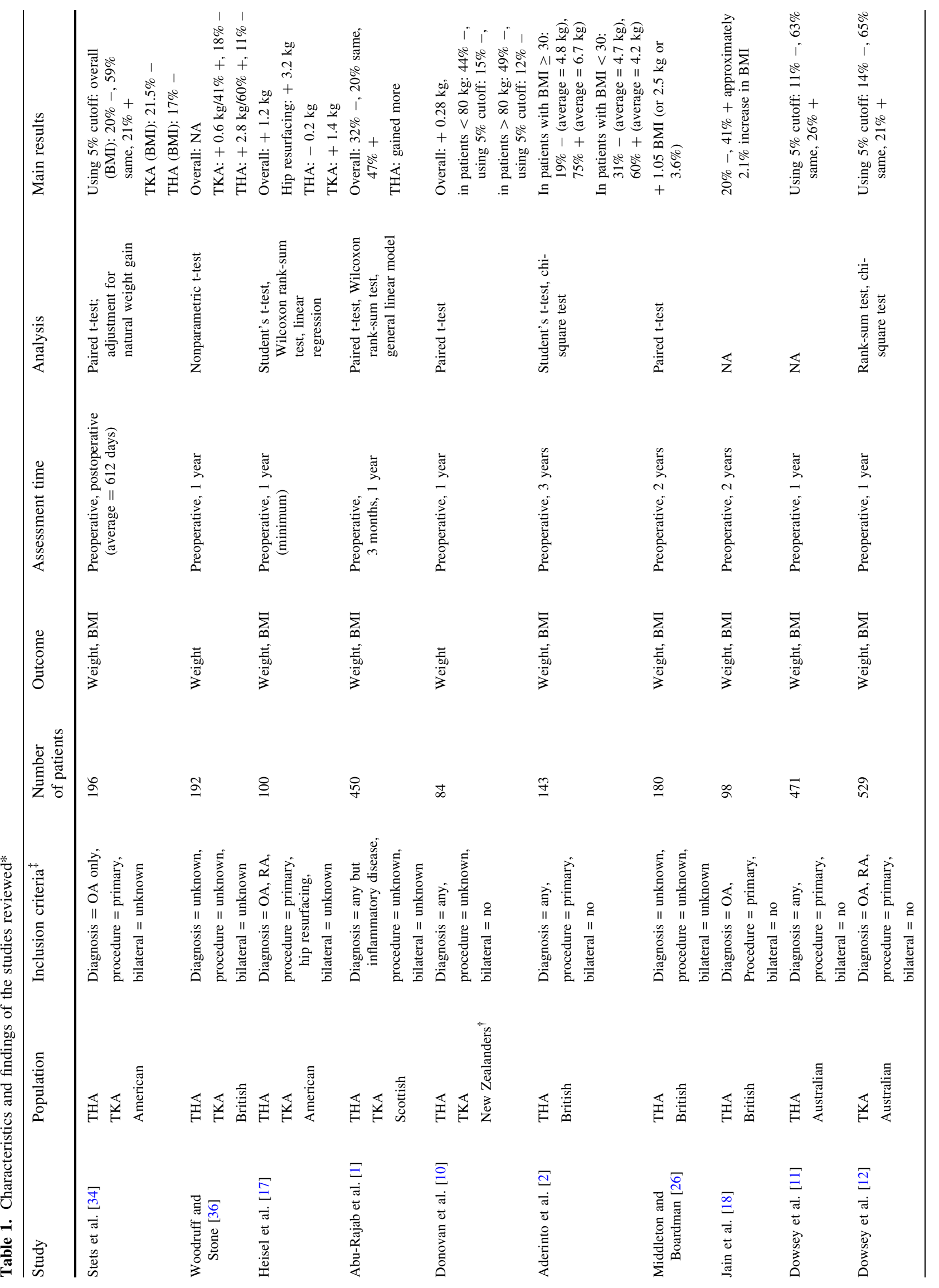




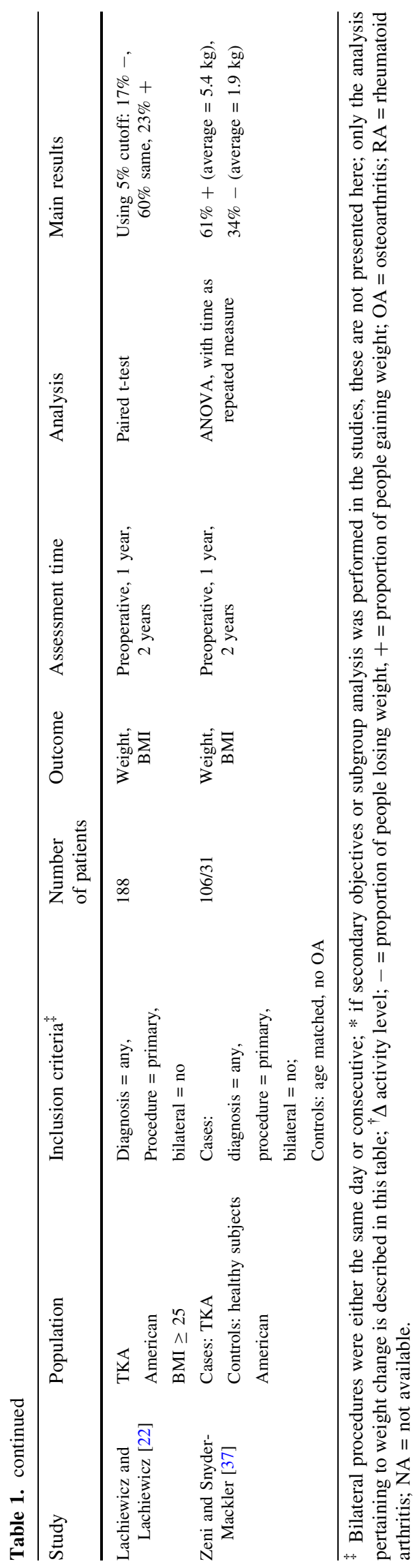

according to the criteria presented by the GRADE $[5,15]$. The quality of evidence was defined as high, moderate, low, and very low. Definitions are based on the design of the study (randomized control trial $=$ high, observational study $=$ low), and then the studies are further assessed based on the limitations, inconsistencies, and uncertainty of the directness of evidence. The limitations of the studies were assessed by evaluating whether the study had appropriate eligibility criteria (selection bias), accurately measured and reported exposure and outcome (detection bias), adequately addressed confounding, and had adequate followup (attrition bias) (Table 2). If no serious limitations were found for each of the criteria, the study was kept at the level first assessed based on design. If high risk of bias existed in any of the criteria examined, its score was reduced by one grade.

Of the 12 studies [1, 2, 10-12, 17, 18, 22, 26, 34, 36, 37], one was a case-cohort study [37] and the other 11 were case series, with the majority from series of single surgeons $(\mathrm{n}=2)[17,22]$ or single hospitals $(\mathrm{n}=6)[11,12$, $18,26,34,37]$. Four studies did not mention the setting or number of surgeons participating in the study $[1,2,10,36]$. Five of the studies included THAs and TKAs, four included only THAs, and three included only TKAs. The patient samples were from the United States $(n=4)$, United Kingdom $(n=5)$, Australia $(n=2)$, and New Zealand $(n=1)$. The average age of patients reported for the samples ranged between 65 to 71 years for 11 studies. One study had a younger sample, with a mean patient age of 58 years (range, 23-82 years) [17]. No study suggested or mentioned that their patients were advised to lose weight or follow any specific diet plan after their TJA as part of the study.

Eligibility criteria and case mix was provided in most, but not all, studies. Studies with stricter inclusion criteria focused on one type of procedure (primary TJA), for osteoarthritis, and excluded bilateral procedures. However, two studies did not provide any information on these three main eligibility criteria (diagnosis, procedure type, bilaterality) $[26,36]$. Of the remaining studies, three did not specify whether bilateral procedures were included in their sample [1, 17, 34] and two did not specify the types of procedures included $[1,10]$. When using these three eligibility criteria, five studies were considered at high risk of selection bias [1, 17, 34, 36, 37], one was moderate risk [22], three were low risk $[11,12,18]$, and the risk could not be evaluated in the three remaining studies [2, 10, 26].

All studies specified that joint arthroplasty was performed (TJA, TKA, and/or THA); however, inclusion of procedures other than primary TJA could not be determined in four studies [1, 10, 26, 36]. All studies were categorized as being low risk of possible detection bias as they included patients with joint arthroplasties. 
Table 2. Summary of study limitations using the GRADE criteria

\begin{tabular}{|c|c|c|c|c|c|}
\hline \multirow[t]{3}{*}{ Study } & \multicolumn{5}{|l|}{ Risk of bias } \\
\hline & \multirow{2}{*}{$\begin{array}{l}\text { Failure to develop and } \\
\text { apply appropriate eligibility } \\
\text { criteria (selection bias) }\end{array}$} & \multicolumn{2}{|c|}{$\begin{array}{l}\text { Flawed measurement of exposure } \\
\text { and outcome (detection bias) }\end{array}$} & \multirow[t]{2}{*}{$\begin{array}{l}\text { Failure to adequately } \\
\text { control confounding }\end{array}$} & \multirow{2}{*}{$\begin{array}{l}\text { Incomplete followup } \\
\text { (attrition bias) (loss } \\
\text { to followup) }\end{array}$} \\
\hline & & $\begin{array}{l}\text { Exposure (joint } \\
\text { arthroplasty) }\end{array}$ & $\begin{array}{l}\text { Outcome (weight } \\
\text { assessment) }\end{array}$ & & \\
\hline Stets et al. [34] & High & Low & High & High & Low $(0 \%)$ \\
\hline $\begin{array}{l}\text { Woodruff and Stone } \\
\text { [36] }\end{array}$ & High & Low & Unclear & High & Low $(0 \%)$ \\
\hline Heisel et al. [17] & High & Low & Unclear & High & Low $(0 \%)$ \\
\hline Abu-Rajab et al. [1] & High & Low & Low & Low & Low $(0 \%)$ \\
\hline Donovan et al. [10] & Unclear & Low & Unclear & High & High $(27 \%)$ \\
\hline Aderinto et al. [2] & Unclear & Low & Unclear & High & Low $(2.1 \%)$ \\
\hline $\begin{array}{l}\text { Middleton and } \\
\text { Boardman [26] }\end{array}$ & Unclear & Low & Unclear & High & Low $(0 \%)$ \\
\hline Jain et al. [18] & Low & Low & High & High & High (20\%) \\
\hline Dowsey et al. [11] & Low & Low & Low & High & Low $(1.5 \%)$ \\
\hline Dowsey et al. [12] & Low & Low & Low & High & Low $(1.5 \%)$ \\
\hline $\begin{array}{l}\text { Lachiewicz and } \\
\text { Lachiewicz [22] }\end{array}$ & Moderate & Low & Low & Moderate & High (19\%) \\
\hline $\begin{array}{l}\text { Zeni and Snyder- } \\
\text { Mackler [37] }\end{array}$ & High & Low & Low & High & Low $(0 \%)$ \\
\hline
\end{tabular}

GRADE $=$ Grading of Recommendations Assessment, Development, and Evaluation .

Weight change was reported differently in studies. The proportion of patients who lost or gained weight (or remained unchanged) was reported in nine studies $[1,2$, $10-12,18,22,34,36,37]$. The remaining two studies only reported overall body composition changes [17, 26]. A low risk of detection bias was assigned if measurements were made in the physicians' office preoperatively and at followup. The protocol for weight or BMI assessment was available in only five studies, which were considered to be of low risk of detection bias $[1,11,12,22,37]$. In two studies, self-reported weights were used [18, 34]; owing to the lack of objective measurement, these studies were categorized at high risk of detection bias. Five studies did not provide any information on the protocol for weight measurement and thus assessment of detection bias was considered unclear $[2,10,17,26,36]$.

Only one study reported adjustment for confounding [1]. Abu-Rajab et al. [1] adjusted multivariable models for sex, age, and type of operation (whether THA or TKA). One study was considered at moderate risk as the authors performed some analyses stratified by sex [22]. No other studies addressed the possibility of confounding by sex, age at the time of surgery, general health status, type of procedure performed, or other known variables associated with the ability of patients to recover from the joint arthroplasty and weight change.

Most studies were considered at low risk of attrition bias as their loss to followup rates were within $0 \%$ to $2.1 \%$ [1,
$2,11,12,17,26,34,36,37]$. Only three studies were considered at high risk of attrition bias, owing to loss to followup ranging between $19 \%$ and $27 \%$ [10, 18, 22].

Because study designs were different, the analyses performed differed. Appropriateness of the analysis was evaluated to ensure results were representative of the study sample. In two studies, no description of the analysis performed for the weight and BMI comparison was available $[11,18]$. In four studies, the nature of the paired data was ignored and proper paired data analysis was not performed $[2,12,17,36]$.

\section{Results}

Owing to the observational nature of these case series and case-cohort studies and the serious limitations found in each study, all studies were considered very low quality according to GRADE criteria.

The overall reported results of the 12 studies, independent of the sample included, type of assessment, and time, show the proportion of patients who lost weight at least 1 year postsurgery ranged from $14 \%$ to $49 \%$.

In five studies, any amount of weight change was used to categorize whether the patient lost or gained weight. Overall, more patients seemed to have gained weight than lost weight according to these studies. The highest proportion of (any) weight loss reported in studies, not using the $5 \%$ body weight change criteria, was reported by 
Donovan et al. [10] for patients who had a preoperative weight greater than $80 \mathrm{~kg}$; however, using the criteria, only $12 \%$ of the patients lost weight. Zeni and Snyder-Mackler et al. [37] reported the next largest proportion of weight loss, followed by Abu-Raja et al. [1], and Aderinto et al. [2] in patients with preoperative BMIs less than $30 \mathrm{~kg} / \mathrm{m}^{2}$. The study reporting the lowest proportion of patients with weight loss postoperatively was Jain et al. [18]. The study reporting the largest proportion of patients gaining weight was Aderinto et al. [2]. Their study, however, had the longest followup of all studies, with the assessment performed 3 years postoperatively. The next highest proportions of patients gaining weight were reported by Zeni and SnyderMackler [37]), Woodruff and Stone [36], Abu-Rajab et al. [1], and Jain et al. [18].

Five studies used the stricter FDA recommendations to define clinically meaningful weight loss (body weight change of 5\%) [14]. Dowsey et al. [11, 12] (in the knee and the hip studies), Donovan et al. [10], and Lachiewicz and Lachiewicz [22] all reported similar results; a range of $11 \%$ to $17 \%$ of patients lost at least $5 \%$ of their body weight within the study followup time. However, Donovan et al. [10] studied a combination of patients who had TKAs and THA, whereas Lachiewicz and Lachiewicz [22] reported on patients who had TKAs only. Stets et al. [34], examining patients who had TKAs and THAs, found $17 \%$ of patients had 5\% body weight loss, but when corrected for natural weight gain the proportion was $20 \%$.

Heisel et al. [17] and Middleton and Boardman [26], did not use the $5 \%$ body weight change criteria and did not categorize patients into groups who lost or gained weight. They reported mean weight changes; Heisel et al. [17] reported a $-0.2-\mathrm{kg}$ change in patients with THA and Middleton and Boardman [26] reported a $2.5-\mathrm{kg}$ gain. Woodruff and Stone [36] reported an increase of $2.8 \mathrm{~kg}$ after THA, which is similar to that reported by Middleton and Boardman [26].

\section{Discussion}

The published studies describing trends of weight and/or body composition patterns after TJA are inconsistent, partially owing to the heterogeneity of the methods used. Data from different samples, with varying selection criteria, different outcomes, and different quality of evidence, are available, and without reviewing all studies together, the correct assessment of the weight trend after TJA is difficult to determine. In this systematic review, we (1) evaluated the quality of evidence of current published literature on postoperative weight trends in patients with TJAs; (2) summarized whether patients with TJAs lose weight after surgery, and (3) summarized the literature regarding whether patients lose a clinically meaningful amount of weight after TJA.

Readers are cautioned about limitations in the literature and some specific to our study. First, the 11 case series and one case-cohort study evaluated in this review do not adequately characterize the general population with TJAs. All studies had small sample sizes and were limited by other inherent biases owing to their study design (ie, possibility of selection bias and attrition bias) and other methodologic issues (ie, possibility of informational bias attributable to lack of confounding adjustment, measurement bias). In the United States, more than 700,000 joint arthroplasties are performed each year [3]; in other highvolume countries, such as the United Kingdom and Australia, approximately 163,000 and 82,000 are performed per year, respectively $[4,28]$. Although the studies included in this review are observational and limited by their design features (ie, lack of randomization, lack of comparison groups, etc), realistically only such a design would be able to characterize a representative sample of the population with TJAs. Although an observational study still would be considered of low quality according to our chosen quality grading, it would be feasible, affordable, and desirable to characterize this large cohort. The studies we reviewed focused mainly on patients in single-center and single-surgeon series. Assessment of nationally representative samples such as the Healthcare Cost and Utilization Project Nationwide Inpatient Sample or large joint arthroplasty registry cohorts would be desirable, but even they would have limitations. For example, the Nationwide Inpatient Sample does not have anthropometric measures of its patients and focuses on an older population, excluding or severely limiting the ability to generalize the results and draw inferences. The American Joint Replacement Registry is currently under development; its data will not be available for some time. Second, our review arguably is limited by the inclusion of heterogeneous samples from the studies. THAs and TKAs were assessed together in some studies; different lengths of followup are presented in the study, and a different mix of diagnoses leading to the procedure make up the samples included in the studies discussed. However, more rigorous inclusion criteria for studies in this review would render us unable to summarize the current literature in the topic. Third, our review is limited by the search criteria used to identify studies and possible publication bias. Only English language manuscripts were reviewed, which could result in the exclusion of other studies on this topic published in other languages. In addition, only published reports were included and therefore this study could suffer from publication bias if other studies have not been published yet or are in the process of being published. However, we included relevant studies we found on the topic (regardless of type, quality, 
and population), the use of recommended criteria for reporting (PRISMA), and assessment of the evidence quality (GRADE).

The evidence available to evaluate the weight trends of patients undergoing joint arthroplasty is limited by the small sample sizes of the studies and the lack of rigor in their methodology, therefore, the low quality of the studies included in this review. Based on a review of the 12 studies on the topic of weight patterns after joint arthroplasty, we found no conclusive evidence that weight or body composition increases, decreases, or remains the same after joint arthroplasties. This lack of evidence is for the more relaxed term of any weight changes and the stricter definitions of clinically meaningful weight changes. Inconclusive systematic reviews, such as ours, highlight gaps in the literature and areas where clinical uncertainty still prevails. We hope that the inability to answer the question regarding patients' weight patterns after joint arthroplasties proposed by this review will stimulate further research in the area.

Although weight reduction is recommended for patients undergoing joint arthroplasty, it is still unclear whether TJA can assist with weight reduction. Larger cohorts could yield adequate statistical power to detect small but clinically meaningful weight changes. In addition, larger samples might permit subgroup analysis to identify patients with a higher chance of losing weight or possibly gaining weight. However, large-scale studies are lacking on this topic. We recommend larger cohorts of patients using stricter methodology for weight assessment to better characterize the natural developments in weight changes in patients undergoing TJA. Some large cohorts of patients who had joint arthroplasties are followed in the US with a large degree of success, although with other primary outcomes, they could provide the opportunity for conducting this study. These cohorts are those followed by welldesigned longitudinal studies or well-structured, large representative TJA registries.

\section{References}

1. Abu-Rajab RB, Findlay H, Young D, Jones B, Ingram R. Weight changes following lower limb arthroplasty: a prospective observational study. Scott Med J. 2009;54:26-28.

2. Aderinto J, Brenkel IJ, Chan P. Weight change following total hip replacement: a comparison of obese and non-obese patients. Surgeon. 2005;3:269-272, 305.

3. Agency for Healthcare Research and Quality. Statistical Brief \#34. Merrill C, Elixhauser A. Hospital Stays Involving Musculoskeletal Procedures, 1997-2005. Available at: http://www.hcup-us.ahrq. gov/reports/statbriefs/sb34.pdf. Accessed May 14, 2010.

4. Australian Orthopedic Association. National Joint Replacement Registry. Hip and Knee Arthroplasty. Annual Report 2010. Available at: http://www.dmac.adelaide.edu.au/aoanjrr/documents/ aoanjrrreport_2010.pdf. Accessed July 7, 2011.
5. Balshem H, Helfand M, Schunemann HJ, Oxman AD, Kunz R, Brozek J, Vist GE, Falck-Ytter Y, Meerpohl J, Norris S, Guyatt GH. GRADE guidelines: 3 . Rating the quality of evidence. J Clin Epidemiol. 2011;64:401-406.

6. Birrell F, Johnell O, Silman A. Projecting the need for hip replacement over the next three decades: influence of changing demography and threshold for surgery. Ann Rheum Dis. 1999; 58:569-572.

7. Blagojevic M, Jinks C, Jeffery A, Jordan KP. Risk factors for onset of osteoarthritis of the knee in older adults: a systematic review and meta-analysis. Osteoarthritis Cartilage. 2010;18:24-33.

8. Cram P, Lu X, Kaboli PJ, Vaughan-Sarrazin MS, Cai X, Wolf $\mathrm{BR}, \mathrm{Li}$ Y. Clinical characteristics and outcomes of Medicare patients undergoing total hip arthroplasty, 1991-2008. JAMA. 2011;305:1560-1567.

9. Dixon T, Shaw M, Ebrahim S, Dieppe P. Trends in hip and knee joint replacement: socioeconomic inequalities and projections of need. Ann Rheum Dis. 2004;63:825-830.

10. Donovan J, Dingwall I, McChesney S. Weight change 1 year following total knee or hip arthroplasty. ANZ J Surg. 2006;76: 222-225.

11. Dowsey MM, Liew D, Stoney JD, Choong PF. The impact of obesity on weight change and outcomes at 12 months in patients undergoing total hip arthroplasty. Med J Aust. 2010;193:17-21.

12. Dowsey MM, Liew D, Stoney JD, Choong PF. The impact of preoperative obesity on weight change and outcome in total knee replacement: a prospective study of 529 consecutive patients. J Bone Joint Surg Br. 2010;92:513-520.

13. Flugsrud GB, Nordsletten L, Espehaug B, Havelin LI, Engeland A, Meyer HE. The impact of body mass index on later total hip arthroplasty for primary osteoarthritis: a cohort study in 1.2 million persons. Arthritis Rheum. 2006;54:802-807.

14. Food and Drug Administration. Center for Drug Evaluation and Research. Guidance for Industry: Developing Products for Weight Management. Available at: http://www.fda.gov/downloads/ Drugs/GuidanceComplianceRegulatoryInformation/Guidances/ucm 071612.pdf. Accessed February 12, 2012.

15. Guyatt GH, Oxman AD, Vist G, Kunz R, Brozek J, AlonsoCoello P, Montori V, Akl EA, Djulbegovic B, Falck-Ytter Y, Norris SL, Williams JW Jr, Atkins D, Meerpohl J, Schünemann HJ. GRADE guidelines: 4. Rating the quality of evidence-study limitations (risk of bias). J Clin Epidemiol. 2011;64:407-415.

16. Harms S, Larson R, Sahmoun AE, Beal JR. Obesity increases the likelihood of total joint replacement surgery among younger adults. Int Orthop. 2007;31:23-26.

17. Heisel C, Silva M, dela Rosa MA, Schmalzried TP. The effects of lower-extremity total joint replacement for arthritis on obesity. Orthopedics. 2005;28:157-159.

18. Jain SA, Roach RT, Travlos J. Changes in body mass index following primary elective total hip arthroplasty: correlation with outcome at 2 years. Acta Orthop Belg. 2003;69:421-425.

19. Kurtz S, Ong K, Lau E, Mowat F, Halpern M. Projections of primary and revision hip and knee arthroplasty in the United States from 2005 to 2030. J Bone Joint Surg Am. 2007;89: 780-785.

20. Kurtz SM, Lau E, Ong K, Zhao K, Kelly M, Bozic KJ. Future young patient demand for primary and revision joint replacement: national projections from 2010 to 2030. Clin Orthop Relat Res. 2009;467:2606-2612.

21. Kurtz SM, Ong KL, Lau E, Bozic KJ, Berry D, Parvizi J. Prosthetic joint infection risk after TKA in the Medicare population. Clin Orthop Relat Res. 2010;468:52-56.

22. Lachiewicz AM, Lachiewicz PF. Weight and activity change in overweight and obese patients after primary total knee arthroplasty. J Arthroplasty. 2008;23:33-40. 
23. Mantilla CB, Horlocker TT, Schroeder DR, Berry DJ, Brown DL. Risk factors for clinically relevant pulmonary embolism and deep venous thrombosis in patients undergoing primary hip or knee arthroplasty. Anesthesiology. 2003;99:552-560; discussion 5A.

24. McMahon M, Block JA. The risk of contralateral total knee arthroplasty after knee replacement for osteoarthritis. J Rheumatol. 2003;30:1822-1824.

25. Memtsoudis SG, Della Valle AG, Besculides MC, Gaber L, Laskin R. Trends in demographics, comorbidity profiles, in-hospital complications and mortality associated with primary knee arthroplasty. J Arthroplasty. 2009;24:518-527.

26. Middleton FR, Boardman DR. Total hip arthroplasty does not aid weight loss. Ann R Coll Surg Engl. 2007;89:288-291.

27. Moher D, Liberati A, Tetzlaff J, Altman DG; PRISMA Group. Preferred reporting items for systematic reviews and metaanalyses: the PRISMA statement. BMJ. 2009;339:b2535.

28. National Joint Registry for England and Wales. 7th Annual Report 2010. Available at: http://www.njrcentre.org.uk/NjrCentre/LinkClick. aspx ?fileticket $=Q k P I 7 k k 6 B 2 E \% 3 \mathrm{~d} \&$ tabid $=86 \& \mathrm{mid}=523$. Accessed July 6, 2011.

29. Ong KL, Kurtz SM, Lau E, Bozic KJ, Berry DJ, Parvizi J. Prosthetic joint infection risk after total hip arthroplasty in the Medicare population. J Arthroplasty. 2009;24(6 suppl):105-109.

30. Sadr Azodi O, Adami J, Lindstrom D, Eriksson KO, Wladis A, Bellocco R. High body mass index is associated with increased risk of implant dislocation following primary total hip replacement: 2,106 patients followed for up to 8 years. Acta Orthop. 2008;79:141-147.

31. Samson AJ, Mercer GE, Campbell DG. Total knee replacement in the morbidly obese: a literature review. ANZ J Surg. 2010; 80:595-599.

32. Santaguida PL, Hawker GA, Hudak PL, Glazier R, Mahomed NN, Kreder HJ, Coyte PC, Wright JG. Patient characteristics affecting the prognosis of total hip and knee joint arthroplasty: a systematic review. Can J Surg. 2008;51:428-436.

33. Skytta ET, Jarkko L, Antti E, Huhtala H, Ville R. Increasing incidence of hip arthroplasty for primary osteoarthritis in 30- to 59-year-old patients. Acta Orthop. 2011;82:1-5.

34. Stets K, Koehler SM, Bronson W, Chen M, Yang K, Bronson M. Weight and body mass index change after total joint arthroplasty. Orthopedics. 2010;33:386.

35. Sturmer T, Gunther KP, Brenner H. Obesity, overweight and patterns of osteoarthritis: the Ulm Osteoarthritis Study. J Clin Epidemiol. 2000;53:307-313.

36. Woodruff MJ, Stone MH. Comparison of weight changes after total hip or knee arthroplasty. J Arthroplasty. 2001;16:22-24.

37. Zeni JA Jr, Snyder-Mackler L. Most patients gain weight in the 2 years after total knee arthroplasty: comparison to a healthy control group. Osteoarthritis Cartilage. 2010;18:510514. 